\title{
OS EFEITOS DA PRODUTIVIDADE E ESCALA NO DESEMPENHO FINANCEIRO DAS EMPRESAS DE TECNOLOGIA DE INFORMAÇÃO
}

\section{THE EFFECTS OF PRODUCTIVITY AND SCALE ON THE FINANCIAL PERFORMANCE OF INFORMATION TECHNOLOGY COMPANIES}

\section{LUIZ ARTUR LEDUR BRITO}

Doutor em Administração de Empresas pela Escola de Administração de Empresas de São Paulo/Fundação Getulio Vargas (EAESP/FGV).

Professor Adjunto da Escola de Administração de Empresas de São Paulo/Fundação Getulio Vargas (EAESP/FGV).

Rua ltapeva, 474, $8^{\circ}$ andar, POI, Bela Vista São Paulo - SP - CEP 01332-000

E-mail: lviz.brito@fgv.br

\section{FERNANDO COELHO MARTINS FERREIRA}

Mestre em Administração de Empresas pela Escola de Administração de Empresas de São Paulo/Fundação Getulio Vargas (EAESP/FGV).

Professor Assistente do Instituto Presbiteriano Mackenzie (UPM). Rua da Consolação, 930, Edifício João Calvino, sala 205, Consolação São Paulo - SP - CEP 01302-090

E-mail:fferreira@mackenzie.br 


\section{RESUMO}

As empresas da nova economia são realmente diferentes? Este trabalho tem como objetivo investigar a estrutura de variabilidade do desempenho financeiro das empresas de tecnologia de informação (TI) no Brasil e determinar a relação entre o desempenho financeiro e dois fatores específicos à empresa: economias de escala e produtividade. Foram utilizados dados secundários sobre as duzentas maiores firmas de TI do Brasil nos anos de 2000 a 2004. A análise utilizou as técnicas de componentes de variância e regressão múltipla. A estrutura de variabilidade do desempenho foi muito similar às empresas de outros setores, indicando uma predominância do efeito empresa individual e limitados efeitos ano e setor. Esses achados podem levantar um questionamento sobre a "distinção" da nova economia. Os resultados também indicaram um efeito significativo da produtividade sobre o desempenho, mas o mesmo não se observa na escala. Discute-se a significância prática da relação entre produtividade e desempenho.

\section{PALAVRAS-CHAVE}

Nova economia; Tecnologia de informação; Produtividade; Escala; Desempenho financeiro.

\section{ABSTRACT}

Are new economy companies really different? This papers aims at investigating the structure of variability in the financial performance of information technology (IT) companies in Brazil, and also at determining the relation between financial performance and two factors specific to companies, namely economies of scale and productivity. Secondary data of the 200 biggest IT companies of Brazil, referring to years 2000 to 2004 , was used. The analysis used the techniques of components of variance and multiple regression. The structure of variability of performance was very similar to companies of other sectors, indicating the predominance of the 
individual firm effect and limited sector and year effects. These findings might raise a question over the "distinction" of the new economy. The results also indicated a significative effect of producitvity on performance, but the same could not be observed on scale. The practical significance of the relation between productivity and performance is discussed.

\section{KEYWORDS}

New economy; Information technology; Productivity; Scale; Financial performance.

\section{INTRODUÇ ̃̃O}

A importância da nova economia para a competitividade das nações é um ponto freqüentemente abordado pela comunidade acadêmica e não-acadêmica. A atenção dada ao setor da tecnologia de informação (TI) justifica-se, no caso do cenário brasileiro, pela sua crescente participação no PIB brasileiro, assim como as suas elevadas taxas de crescimento observadas desde a década de I990, mais notadamente após o fim da reserva de informática, conforme informa o Ministério da Ciência e Tecnologia (MCT, 2003). Estes, inclusive, são alguns dos fatores que levaram a atual administração federal a incluir o setor de TI em sua política industrial, com a adoção de medidas que visam aumentar a sua competitividade e, por conseguinte, inserção no mercado internacional, conforme informa o Ministério do Desenvolvimento Indústria e Comércio Exterior (MDIC, 2004).

O destaque que o setor de TI tem adquirido, contudo, levanta a questão de até que ponto os fatores que podem determinar o desempenho das firmas desse setor são diferentes daqueles da agora chamada economia tradicional, ou seja, quais são os componentes, ou estrutura, do desempenho financeiro dessa indústria. McGahan e Porter (I997) identificaram que a estrutura de variabilidade poderia ser bastante diferente em diversos setores econômicos. O setor de TI não foi ainda explorado individualmente, embora exista toda uma discussão tanto na academia como na imprensa de divulgação sobre a distinção da nova economia e de um novo padrão competitivo.

Este trabalho tem dois objetivos relacionados entre si. O primeiro é determinar a estrutura de variabilidade do desempenho das empresas de TI, no Brasil, e comparar essa estrutura com aquela encontrada para outras empresas de setores tradicionais no Brasil e no exterior. O segundo objetivo é avaliar o impacto no desempenho de dois conceitos-chave de operações, ligados à empresa individual: economia de escala e nível de produtividade da operação. 
Os resultados não mostram evidências de que a nova economia seja diferente, pelo menos quando se avalia a estrutura de variabilidade do desempenho financeiro. Os resultados mostraram uma estrutura de variância muito similar aos estudos anteriores realizados no Brasil e no exterior com empresas de setores tradicionais. Com relação ao segundo objetivo, foram encontradas evidências claras de uma relação positiva entre produtividade e desempenho, mas não foi possível encontrar efeitos de escala ou escopo influindo no desempenho financeiro.

O trabalho está divido da seguinte forma: após a introdução deste estudo, apresenta-se a sua revisão de literatura pertinente, enfocando os estudos mais influentes sobre a variabilidade no desempenho financeiro e aqueles relacionados aos efeitos da escala e da produtividade sobre o desempenho das firmas; em seguida, apresenta-se a metodologia do trabalho, demonstrando-se como foram obtidos os dados necessários para a nossa análise e a necessária operacionalização das variáveis; uma vez expostos os métodos adotados, parte-se para a apresentação e discussão dos resultados, expondo-se as descobertas mais fundamentais; por fim, procede-se à conclusão, destacando-se as principais implicações deste trabalho e suas evidentes limitações.

\section{REFERENCIAL TEÓRICO \\ 2.1 A DECOMPOSIÇÃO DA VARIABILIDADE NO DESEMPENHO FINANCEIRO}

Esta linha de pesquisa começou realmente com o trabalho de Rumelt (I99I), que foi o primeiro pesquisador a aplicar a técnica de componentes de variância à variabilidade do desempenho das empresas. Seu trabalho ampliou consideravelmente o trabalho inicial de Schmalensee (I985) e conseguiu identificar o efeito empresa, ou seja, a parte da variância que podia ser associada à empresa individual. Vários outros pesquisadores ampliaram o trabalho de Rumelt (I99I), incorporando outros efeitos e explorando amostras mais amplas (ROQUEBERT; PHILLIPS; WESTFALL, I996; BRUSH; BROMILEY, I997; MCGAHAN; PORTER, I997; BRUSH; BROMILEY; HENDRICKX, I999; MCNAMARA; VAALER; DEVERS, 2003).

Os resultados apresentaram grande consistência, indicando que o efeito mais importante, ou o que tinha potencial de explicar o maior percentual da variância observada, era o efeito empresa individual. O desempenho estava prioritariamente associado a características intrínsecas e idiossincráticas das empresas. Os valores variaram, mas, em geral, a empresa individual foi capaz 
de explicar cerca de $40 \%$ da variância total, e o percentual total de variância explicada ficava em torno de $60 \%$. Um dos fatores intrigantes foi especificamente o baixo percentual de variância associada ao fator ano que não passava de alguns pontos percentuais.

Esses resultados foram usados para suportar a visão baseada em recursos da estratégia (WERNERFELT, I984; BARNEY, I99I; BARNEY; ARIKAN, 200I) em oposição à escola de posicionamento liderada por Porter (I985).

No Brasil, investigações sobre empresas brasileiras indicaram uma composição de variância muito semelhante, novamente com um efeito ano pequeno, apesar das instabilidades da economia (BRITO; VASCONCELOS, 2004; GONÇALVES; QUINTELLA, 2005).

Nenhuma dessas investigações explorou, contudo, a realidade de empresas atuantes na nova economia. A natureza desse setor emergente, com mudanças tecnológicas contínuas, poderia teoricamente fazer supor uma estrutura mais dependente do fator ano e possivelmente menos previsível dado o dinamismo do setor. Essa investigação é uma das contribuições deste estudo.

\subsection{ESCALA E PRODUTIVIDADE COMO FATORES QUE CONTRIBUEM PARA O DESEMPENHO DAS EMPRESAS}

\subsubsection{Tamanho e economias de escala e escopo}

As economias de escala, justificadas pela indivisibilidade dos custos fixos pela impossibilidade de ter unidades menores que um determinado tamanho, é um tema freqüente em operações. A indivisibilidade é mais provável quando a produção usa plantas que requerem elevados investimentos de capital, e a economia de escala em serviços pode não ser tão evidente. Outras fontes de economia de escala são: o aumento da produtividade dos insumos variáveis; inventários; ganhos na operação de compra, propaganda, distribuição e pesquisa e desenvolvimento (BESANKO et al., 2004).

Relacionada à economia de escala está a economia de escopo. Esse segundo tipo de economia é resultante da produção conjunta de uma variedade de produtos que apresentam algum tipo de sinergia. Nesse caso, a soma dos custos de produção dos bens em firmas separadas é maior que a produção conjunto em uma única firma. Os custos são incorridos em conjunto para a produção de unidades para diversos negócios. Economias de escopo podem ser tratadas como uma modalidade de economia de escala, pois ela aumenta o tamanho da firma e as fontes de economia de escala anteriormente apontadas podem ser associadas às economias de escopo (PORTER, I996; BESANKO et al., 2004). 
Algumas deseconomias de escala são apontadas na literatura. Besanko et al. (2004) apontam algumas fontes principais de deseconomias de escala: custo de salários; efeitos da burocracia; divisão de recursos especializados; transferência de informação entre clientes; e conflito de interesses entre stakeholders. Ricketts (2002) também aponta problemas de deseconomias de escala associados a problemas de coordenação decorrentes da complexidade da firma maior.

Zenger (I994), por exemplo, estudou as razões pelas quais pequenas firmas freqüentemente apresentavam vantagens em eficiência de pesquisa e desenvolvimento, e acabavam sendo mais bem-sucedidas do que grandes corporações. Os problemas de agência e as deseconomias de escala decorrentes desses problemas eram comuns, sendo as firmas menores mais eficientes em resolvê-los. No entanto, Henderson e Cockburn (I996) argumentam no sentido oposto, identificando economias de escala e escopo em P\&D no setor farmacêutico, concluindo que as empresas maiores têm vantagens em relação às empresas menores.

Capon, Farley e Hoenig (1990) apresentaram um trabalho que cobriu vários fatores que afetavam potencialmente o desempenho financeiro e entre eles o tamanho. Os autores revisaram 320 estudos empíricos publicados entre I92I e I987. Desse total de estudos, 67 investigaram especificamente a relação entre tamanho e desempenho financeiro. A conclusão da análise é que não é possível afirmar que a relação seja significativa. Como afirmam os autores: "O tamanho, por si só, não confere lucratividade.” (CAPON; FARLEY; HOENIG, I990, p. II57, tradução nossa). Uma segunda revisão foi a de Gooding e Wagner III (I985) que analisaram com outra metodologia e grau maior de profundidade 3I estudos empíricos específicos sobre tamanho e desempenho, e indicaram que as pesquisas empíricas, quando tomadas de forma geral, apresentavam resultados contraditórios e não-conclusivos. A conclusão a que os autores puderam chegar é que não havia evidências que pudessem indicar qualquer relação entre o tamanho e o desempenho quando tomado de forma relativa.

Se os resultados quanto à existência de economias de escala e escopo líquidas para empresas de manufatura é uma questão não resolvida, mais se justifica a dúvida com relação a empresas de serviço. O setor objeto desta pesquisa é composto por empresas que têm um componente de serviço relativamente alto no seu negócio.

\subsubsection{Produtividade e desempenho}

As medidas de produtividade não são poucas e podem variar bastante em razão das características de uma indústria (HOQUE; FALK, 2000). De maneira geral, a produtividade pode ser definida como a razão entre uma medida de 
output e a medida de algum ou de todos os recursos utilizados para produzir esse output. Essa definição, apesar de soar intuitivamente correta, parece não abarcar toda a complexidade por trás dos índices de produtividade das firmas. Diversos estudos, por exemplo, calculam a produtividade a partir da razão entre o output e apenas um dos inputs (ou recursos), como é o caso daqueles que utilizam somente a força de trabalho para calculá-la, sem considerar os insumos e bens de capital utilizados (EASTERFIELD, I965). Outro problema apontado para essa definição é que ela considera apenas a quantidade final produzida como numerador, não levando em consideração a mudança na qualidade dos inputs ou outputs. As medidas de produtividade capturam as economias associadas com o melhor uso dos inputs, mas o aumento de produtividade associado à maior qualidade dos outputs pode não ser capturado (MISTEREK, I992). Iniciativas para refinar, modificar ou melhorar os índices de medição de produtividade já foram realizadas (MILLER, I984, I987; MILLER; RAO, I989).

Independentemente da polêmica em torno desse conceito, a relação entre a produtividade e o desempenho das firmas já foi amplamente estudada pelas ciências econômicas e administração, e podem ser encontradas na literatura evidências tanto a favor de uma relação positiva entre estes elementos (DAVIES; GALLMAN; HUTCHINS, I987; BANKER; CHANG; MAJUNDAR, I993; LA PORTA; LOPEZ-DE-SILANES, I999) como a de uma relação não existente.

Hitt e Brynjolfsson (I996), por exemplo, não encontraram nenhuma relação entre produtividade e desempenho ao estudarem 370 firmas de grande porte. Esses autores, avaliando o impacto dos investimentos em TI nas firmas, verificaram que esses investimentos foram capazes de aumentar a produtividade e gerar maior valor ao consumidor, mas não encontraram evidências de que esses benefícios se converteram em lucros acima da média para as firmas. Já Anderson, Fornell e Rust (1997) sugerem que o aumento da produtividade, não por meio da geração de maior output, mas pela redução de pessoal nos setores intensivos em mão-de-obra (bancário, educacional e hospitalar), pode trazer resultados satisfatórios em curto prazo, mas também pode minar o desempenho desses setores em um período mais longo.

\section{PROCEDIMENTO METODOLÓGICO}

\section{AMOSTRA}

A pesquisa Info 200, publicada anualmente pela revista Exame, que apresenta informações de cunho econômico, financeiro e de produtividade sobre as duzentas maiores empresas de tecnologia de informação do Brasil, foi a fonte dos 
dados utilizada. Foram usadas as últimas cinco edições publicadas da Info 200, referentes aos resultados das empresas desde o ano 2000 até 2004. Essas edições forneceram a amostra inicial de mil observações que cobriram sete ramos de negócios da indústria de tecnologia de informação, a saber: comunicações, hardware, software, infra-estrutura, serviços de software, serviços operacionais e distribuição. Por causa dos processos de fusões e aquisições ocorridos nos últimos anos, principalmente no que diz respeito à telefonia celular, preferiu-se retirar quatro observações da amostra, a fim de evitar distorções nos resultados da análise. Essa amostra não pode ser considerada probabilística, e, portanto, as possibilidades de generalização são limitadas. A amostra é, contudo, relevante, pois o faturamento agregado das empresas nela contidas representou II\% do PIB nacional, no ano de 2004 (EXAME, 2005).

\subsubsection{Desempenho financeiro das empresas}

A operacionalização do desempenho, mesmo que restrita ao aspecto financeiro, apresenta ao pesquisador várias alternativas. Glick, Washburn e Miller (2005) discutem essas múltiplas alternativas e suas dificuldades. Os estudos de componentes de variância também exploraram de forma empírica os efeitos de diferentes formas de operacionalização, concluindo que praticamente todas as formas levam a resultados semelhantes, pelo menos quanto à estrutura de variabilidade dos mesmos (HAWAWINI; SUBRAMANIAN; VERDIN, 2003). Uma distinção importante é entre lucratividade e crescimento que podem apresentar comportamentos diferentes em relação a variáveis e mesmo quanto à estrutura de variabilidade (GEROSKI; MACHIN; WALTERS, I997).

Nesta pesquisa, também se levou em conta as limitações dos dados disponíveis, e o desempenho financeiro foi operacionalizado por três variáveis, calculadas para cada ano-empresa analisado: a razão entre lucro líquido e vendas totais; a razão entre EBITDA (Earnings Before Interest, Tax, Depreciation, and Amortization) e vendas totais; o percentual de crescimento das vendas em relação ao ano anterior, descontada a inflação pelo IGPM. Como todas as operacionalizações são razões, evita-se o problema de unidades. A primeira operacionalização usa o critério contábil mais comum de lucro líquido, levando em conta a carga de impostos e custos financeiros da empresa, sendo uma medida mais ampla de lucratividade. A segunda operacionalização é independente desses fatores e relaciona-se mais diretamente com a capacidade de geração de caixa do negócio. Finalmente, a terceira operacionalização aborda a questão do crescimento. O uso de três operacionalizações diferentes do mesmo construto contribui para a validade deste e permite uma avaliação de validade convergente (KERLINGER; LEE, 2000, p. 670-672). 
Essas operacionalizações do construto desempenho, por basearem-se em dados estritamente contábeis, às vezes apresentam valores causados por situações contábeis específicas que não representam o construto que queremos avaliar. Por exemplo, lucros líquidos maiores (ou menores) que as vendas em um ano específico devem ter sido causados por variações patrimoniais decorrentes de outras variáveis que não a operação propriamente dita. Invocando o critério de validade de construto, eliminamos as observações que apresentavam valores extremos em cada uma das operacionalizações quando da análise destas. Os valores de lucro líquido sobre vendas e EBITDA sobre vendas menores que -Ioo\% ou maiores que ı००\% foram eliminados. Também valores de taxas de crescimento menores que $-50 \%$ e maiores que 100\%, representando empresas que encolheram a menos da metade de seu tamanho no ano anterior, ou que mais que dobraram de tamanho em um ano, foram eliminados.

\subsubsection{Tamanho e potenciais economias de escala e escopo}

A discussão de como operacionalizar tamanho existe há bastante tempo na academia. A noção de tamanho é ampla, envolvendo múltiplas dimensões, já que qualquer operacionalização deixa de lado algum aspecto do construto (PENROSE, I959; GUPTA, I980). As operacionalizações mais comuns na literatura foram o número de empregados, vendas totais e ativos totais (SUTTON, I997). Kimberly (I976) ressalta que a operacionalização do tamanho deve levar em conta o objetivo da pesquisa. Nesse caso, a idéia é usar o tamanho como um indicador de possíveis economias de escala disponíveis à empresa. Novamente, levando-se em conta as opções disponíveis, optou-se por usar as vendas totais como variáveis que representassem o construto tamanho. A alternativa de ativos totais poderia ter limitações, já que muitas empresas têm um componente de serviços elevado. A alternativa de usar o número de empregados poderia combinar efeitos de produtividade, ou seja, aqueles que seriam estudados de forma separada.

As vendas totais foram reajustadas em reais ao ano de 2004 pelo IGPM, para compensar o efeito da inflação. Seguindo a maioria dos estudos, foi usada uma transformação logarítmica dessa variável, com o logaritmo na base ıo das vendas totais em reais de 2004. Kimberly (1976) discutiu as justificativas substantivas e metodológicas dessa transformação. Primeiro, a relação entre o tamanho e as outras variáveis organizacionais não parece ser linear e pode apresentar um efeito multiplicativo em vez de aditivo. Segundo, a transformação evita o problema de tratar uma distribuição muito assimétrica e distante da distribuição normal. 


\subsubsection{Produtividade}

A produtividade pode ser definida como a razão entre um resultado (output) e um insumo (input). No caso dos dados disponíveis, havia poucas possibilidades de escolha. A razão entre as vendas totais e o número de empregados foi selecionada como um indicador geral de produtividade. Espera-se que quanto maior for essa razão, maior será a produtividade da empresa, pelo menos no que tange à mão-de-obra, um insumo muito importante nesse setor. Essa produtividade poderia, porém, ser substancialmente diferente nos vários ramos de negócio analisados, que poderiam demandar intensidades de mão-de-obra distintas. A variável original de vendas por funcionário foi então relativizada em relação à média de cada ramo de negócios e definida como:

$$
\text { Produtividade }=\frac{\text { Vendas por funcionário }}{\text { Média das vendas por funcionário do ramo }}-\mathrm{I}
$$

Os valores de produtividade calculados para cada ramo de negócios estão apresentados na Tabela I.

\section{TABELA I}

PRODUTIVIDADE DOS SETORES DE TI PRESENTES NA AMOSTRA

\begin{tabular}{lccccccc}
\hline & $\begin{array}{l}\text { COMUNI- } \\
\text { CAÇÕES }\end{array}$ & $\begin{array}{c}\text { SERVIÇOS DE } \\
\text { SOFTWARE }\end{array}$ & $\begin{array}{c}\text { SERVIÇOS } \\
\text { OPERACIONAIS }\end{array}$ & SOFTWARE & $\begin{array}{c}\text { INFRA- } \\
\text { ESTRUTURA }\end{array}$ & HARDWARE & DISTRIBUIÇÃo \\
\hline Média & 301,4 & 140,1 & 103,0 & 347,7 & 422,2 & 507,4 & 461,3 \\
\hline Mediana & 237,7 & 65,5 & 49,7 & 184,2 & 247,4 & 216,7 & 317,5 \\
\hline $\begin{array}{l}\text { Desvio } \\
\text { padrão }\end{array}$ & 220,4 & 195,3 & 189,3 & 739,1 & 489,9 & 1097,1 & 737,2 \\
\hline Mínimo & 15,9 & 6,2 & 3,6 & 16,2 & 34,6 & 23,1 & 7,5 \\
\hline Máximo & 994,5 & 1344,3 & 1774,5 & 7500,0 & 2874,1 & 8666,7 & 6044,0 \\
\hline
\end{tabular}

Fonte: Elaborada pelos autores.

\subsubsection{Variáveis de controle}

O primeiro grupo de variáveis de controle teve por objetivo controlar diferenças entre os sete diferentes ramos compreendidos na amostra. Foram criadas seis variáveis dicotômicas (com valor o e I) para isso. O valor zero corresponde 
ao ramo comunicações em todos os casos. As seis variáveis (hardware, software, infra-estrutura, serviços de software, serviços operacionais e distribuição) assumem o valor i quando o ramo diz respeito ao setor correspondente. Dessa forma, efeitos fixos nas variáveis de desempenho comuns a todas as empresas de um determinado ramo são controlados.

O segundo grupo de variáveis de controle teve por objetivo controlar as diferenças entre os diferentes anos quando foram coletados os resultados da amostra. Foram criadas quatro variáveis dicotômicas para tal. O valor o dessas variáveis foi atribuído sempre ao ano 2004. As quatro variáveis (ano 2000, ano 200I, ano 2002 e ano 2003) assumem o valor I quando a observação corresponde ao ano em questão. Assim, os coeficientes dessas variáveis nas regressões expressam o efeito diferencial deste ano em relação ao ano de 2004, no desempenho das empresas.

O terceiro grupo de variáveis de controle teve o objetivo de controlar diferentes formas de governança das empresas. Essas variáveis têm também um interesse intrínseco, e os resultados associados a elas são uma contribuição adicional da pesquisa. O país onde o controle da empresa está sediado foi uma dessas variáveis. Novamente, utilizou-se uma variável dicotômica (controle estrangeiro) com o valor o para controle brasileiro e o valor I para o controle estrangeiro. $O$ fato de a empresa ter capital aberto e ações na bolsa de valores foi a segunda variável de governança. Outra variável dicotômica (ações na Bolsa) com valor o para empresas de capital fechado e i para empresas de capital aberto foi utilizada.

\subsubsection{Procedimentos de análise}

Inicialmente, determinou-se a composição de variância das três operacionalizações do desempenho financeiro usando a técnica de componentes de variância. A rotina de componentes de variância do SPSS I3.0, com o método de estimação Minque, foi utilizada. Essa técnica trata os diversos grupos de fatores que afetam o desempenho como fatores aleatórios e decompõe a variância total em variâncias componentes associadas a cada um desses grupos de fatores (SEARLE; CASELLA; MCCULLOCH, I992). É possível assim identificar o percentual da variância total que é atribuível ao fato de a empresa pertencer a diferentes ramos de negócios (cada um dos sete ramos presentes na amostra é tratado como uma realização desta variável aleatória - ramo de negócios), o percentual atribuível aos diferentes anos, o percentual atribuível às interações ano-ramo, ou seja, situações específicas a um ramo em um ano específico. Estes são os efeitos ramo, ano e interação ano-ramo, respectivamente. Também se avalia o percentual da variância que é atribuível à empresa individual, representando a influência de suas características próprias e idiossincráticas ao seu desempenho. Finalmente, a variância não-atribuível a nenhum desses fatores é a variância não-explicável pelo modelo. Esta é uma análise apenas descritiva, 
mas é possível inferir a relevância de cada grupo de fatores pelo percentual de variância que eles conseguem capturar.

Em uma segunda etapa da análise, três regressões múltiplas (uma para cada operacionalização do desempenho financeiro como variável dependente) foram feitas. Cada regressão teve catorze variáveis independentes (seis variáveis de controle para o ramo de negócios, quatro variáveis para os diferentes anos, duas variáveis para a caracterização do sistema de governança e as duas variáveis de interesse - tamanho e produtividade).

\section{RESULTAdOS E DISCUSSÃO}

\section{ANÁLISE DE COMPONENTES DE VARIÂNCIA}

As três operacionalizações do desempenho financeiro foram, inicialmente, submetidas à análise de componentes de variância. Os resultados dessa análise, bem como as características descritivas das variáveis e das amostras finais sobre as quais foi feita a análise, estão apresentados na Tabela 2. No caso do EBITDA sobre vendas, os dados de 2000 e 200 I não estavam disponíveis, assim o ano teve apenas três valores. Em dois casos, obtiveram-se componentes de variância levemente negativos (o efeito ano no caso de EBITDA sobre vendas e o efeito setor no caso do crescimento das vendas). Nesses casos, seguiu-se a recomendação de atribuir o valor o e recalcular o modelo sem esse fator (SEARLE; CASELLA; MCCULLOCH, I992).

Em todos os casos, confirmando o encontrado em estudos anteriores sobre empresas americanas e brasileiras (MCGAHAN; PORTER, I997; HAWAWINI; SUBRAMANIAN; VERDIN, 2003; BRITO; VASCONCELOS, 2004; GONÇALVES; QUINTELLA, 2005), o efeito empresa individual foi o mais relevante. No caso das operacionalizações de lucro líquido e EBITDA sobre vendas, ele explicou quase a metade da variância total, valor similar ao encontrado nesses estudos anteriores. No caso do crescimento, ele teve um valor menor seguindo também um menor percentual total de variância explicada. A composição da variância da taxa de crescimento foi menos estudada na literatura, mas o valor encontrado é coerente com a análise de Geroski, Machin e Walters (i997) que, com outra metodologia, também encontraram um maior nível de variabilidade não-explicável nas taxas de crescimento.

A pequena influência do fator ano nas duas primeiras operacionalizações do desempenho, apesar de também coerente com os estudos anteriores, não confirma as afirmações de maior volatilidade e dinamismo que poderiam estar associadas a negócios da nova economia. Esse efeito só foi perceptível no caso do crescimento quando o efeito ano e sua interação com o ramo de negócios foram maiores. 


\section{TABELA 2}

ANÁLISE DOS COMPONENTES DE VARIÂNCIA

DAS VARIÁVEIS DEPENDENTES

\begin{tabular}{|c|c|c|c|}
\hline & $\begin{array}{c}\text { LUCRO LÍQUIDO SOBRE } \\
\text { VENDAS }\end{array}$ & $\begin{array}{l}\text { EBITDA SOBRE } \\
\text { VENDAS }\end{array}$ & $\begin{array}{c}\text { CRESCIMENTO DAS } \\
\text { VENDAS }\end{array}$ \\
\hline Média & 1,2 & 10,2 & 7,1 \\
\hline Mediana & 2,9 & 8,3 & 2,9 \\
\hline Desvio padrão & 18,7 & 17,9 & 25,9 \\
\hline Efeito ano & $0,4 \%$ & $0,0 \%$ & $15,7 \%$ \\
\hline $\begin{array}{l}\text { Efeito ramo de } \\
\text { negócios }\end{array}$ & $9,3 \%$ & $9,6 \%$ & $0,0 \%$ \\
\hline Interação ramo x ano & $6,9 \%$ & $6,0 \%$ & $2,8 \%$ \\
\hline Efeito empresa & $48,0 \%$ & $47,2 \%$ & $23,1 \%$ \\
\hline Variância explicada & $64,7 \%$ & $62,8 \%$ & $41,5 \%$ \\
\hline $\begin{array}{l}\text { Variância não- } \\
\text { explicada }\end{array}$ & $35,3 \%$ & $37,2 \%$ & $58,5 \%$ \\
\hline Total & $100,0 \%$ & $100,0 \%$ & $100,0 \%$ \\
\hline Empresas & 271 & 215 & 325 \\
\hline Ramos de negócios & 7 & 7 & 7 \\
\hline Anos & 5 & 3 & 5 \\
\hline Observações & 714 & 439 & 875 \\
\hline
\end{tabular}

Fonte: Elaborada pelos autores.

Quando tomados em conjunto, os resultados de componentes de variância não indicam diferenças marcantes entre a variabilidade do desempenho das empresas de tecnologia de informação e outros setores da economia. Fatores associados à empresa individual são os responsáveis pela maior parcela de variabilidade, indicando que aí deve se concentrar o estudo dos fatores que afetam o desempenho. O restante da análise desta pesquisa tenta justamente explorar mais detalhadamente dois destes fatores: a produtividade de cada empresa e as economias de escala disponíveis a cada uma destas empresas. Essa constatação 
da relevância do efeito empresa individual também indica a aplicabilidade da visão baseada em recursos da estratégia à análise estratégica das empresas do setor de tecnologia da informação.

\subsection{ANÁLISE DAS REGRESSÕES}

Todas as três regressões tendo as diferentes operacionalizações de desempenho como variável dependente tiveram elevada significância estatística com p-valor menor que $0,00 \mathrm{I}$ em todos os casos. O total de variância explicado pelas regressões ficou entre ı०,6\% e I7,0\%. Fica a questão de como avaliar a significância prática desses valores. Isso pode ser feito tomando-se, por exemplo, o caso do lucro líquido sobre vendas. A regressão explicou I7,0\% da variância total, mas a interpretação gerencial deve ser feita em cima do desvio padrão e não da variância diretamente. Na Tabela 2, pode-se verificar que a média do lucro líquido sobre vendas foi de I,2\% e o desvio padrão de I8,6 pontos percentuais. Sendo o desvio padrão a raiz quadrada da variância, o desvio padrão da variância explicada pela regressão seria de 7,7 pontos percentuais (raiz quadrada de 0,17 x I8,62). Sob o ponto de vista gerencial, é fácil apreciar que uma distribuição com 7,7 pontos percentuais de desvio padrão é relevante. Uma empresa que estivesse com apenas um desvio padrão acima da média teria um lucro líquido de $8,9 \%$ sobre vendas, enquanto uma empresas com um desempenho de desvio padrão abaixo da média teria um prejuízo de $6,5 \%$ sobre vendas. É possível, portanto, afirmar que os resultados têm significância estatística e prática.

\section{TABELA 3}

RESULTADOS DA REGRESSÃO PARA AS VARIÁVEIS

DEPENDENTES DA PESQUISA

\begin{tabular}{lccc}
\hline & $\begin{array}{c}\text { LUCRO LÍQUIDO } \\
\text { SOBRE VENDAS }\end{array}$ & $\begin{array}{c}\text { EBITDA SOBRE } \\
\text { VENDAS }\end{array}$ & $\begin{array}{c}\text { CRESCIMENTO DAS } \\
\text { VENDAS }\end{array}$ \\
\hline Coeficiente de determinação $\left(R^{2}\right)$ & 0,170 & 0,125 & 0,106 \\
\hline $\begin{array}{l}\text { Coeficiente de determinação ajustado } \\
\left(R^{2} \text { adj.) }\right.\end{array}$ & 0,153 & 0,100 & 0,091 \\
\hline Distribuição & $18,73^{* * *}$ & $-5,26$ & $-4,30$ \\
\hline Hardware & $16,72^{* * *}$ & $-2,69$ & $-2,50$ \\
\hline Infra-estrutura & $12,26^{* * *}$ & $-11,84^{* * *}$ & $-3,65$ \\
\hline
\end{tabular}




\section{TABELA 3 (CONTINUAÇÃO)}

RESULTADOS DA REGRESSÃO PARA AS VARIÁVEIS

DEPENDENTES DA PESQUISA

\begin{tabular}{lccc}
\hline & $\begin{array}{c}\text { LUCRO LIQUIDO } \\
\text { SOBRE VENDAS }\end{array}$ & $\begin{array}{c}\text { EBITDA SOBRE } \\
\text { VENDAS }\end{array}$ & $\begin{array}{c}\text { CRESCIMENTO DAS } \\
\text { VENDAS }\end{array}$ \\
\hline Software & $21,02^{* * *}$ & 5,17 & $-5,29$ \\
\hline Serviços operacionais & $20,95^{* * *}$ & 4,52 & $-1,00$ \\
\hline Serviços de software & $20,80^{* * *}$ & 2,16 & $-4,84$ \\
\hline Ano 2000 & $-2,70$ & - & $12,80^{* * *}$ \\
\hline Ano 2001 & $-4,67^{* *}$ & - & $5,44^{* *}$ \\
\hline Ano 2002 & $-4,09^{* *}$ & $-1,62$ & $-12,87^{* * *}$ \\
\hline Ano 2003 & $-0,92$ & $-0,38$ & 0,74 \\
\hline Controle estrangeiro & $-6,21^{* * *}$ & $-3,39^{*}$ & $-1,01$ \\
\hline Ações na bolsa & $5,55^{* *}$ & $9,19^{* *}$ & $-3,67$ \\
\hline Produtividade & $1,57^{* *}$ & $1,47^{* * *}$ & $1,86^{* *}$ \\
\hline Tamanho & 1,87 & 1,66 & $-1,33$ \\
\hline
\end{tabular}

Fonte: Elaborada pelos autores.

* Significante ao nível de ı०\%; ${ }^{* *}$ significante ao nível de 5\%; **** significante ao nível de ı\%.

A análise dos coeficientes das variáveis de controle, Tabela 3, oferece um aprofundamento na compreensão da dinâmica do setor. No caso do lucro líquido sobre vendas, todos os coeficientes apresentaram elevada significância estatística e todos foram positivos com valores relativamente próximos na faixa de I2 a 2 I. Isso indica que o ramo de comunicações, selecionado como caso-base, realmente apresenta uma lucratividade sobre vendas claramente inferior aos demais. O mesmo não se manifesta quando analisamos os coeficientes de EBITDA sobre vendas ou o crescimento sobre vendas. Nesse caso, quase todos os coeficientes não apresentaram valores estatisticamente significativos. Com a exceção do ramo de comunicações no que diz respeito ao lucro líquido sobre vendas e do ramo de infra-estrutura no que diz respeito ao EBITDA sobre vendas, não é possível identificar diferenças no desempenho entre os diversos ramos de negócios no nível das empresas individuais. 
A análise dos coeficientes das variáveis ano apresenta um quadro um pouco diferente. Tanto no caso do lucro líquido como no caso do crescimento, vários coeficientes apresentam valores estatisticamente significativos e com valores ora positivos ora negativos. Isso confirma, para o caso do crescimento, o fato já constatado na análise de componentes de variância que indicou uma influência maior desse fator para o caso do crescimento. Em termos de lucratividade, pareceria que 2004 (ano tomado como base) foi um ano bastante favorável para as empresas do setor, já que todos os coeficientes dos demais anos foram negativos, indicando resultados inferiores (200I e 2002 com diferenças significativas). Para o crescimento, 2004 parece ter sido um ano equivalente a 2003 , bem melhor que 2002, mas ainda inferior aos anos de 2000 e 200 I que apresentaram coeficientes positivos e estatisticamente significativos.

As duas variáveis de governança apresentaram coeficientes estatisticamente significativos para as regressões de lucro líquido e EBITDA, mas não para o crescimento. Pode-se dizer que as empresas de controle estrangeiro apresentam lucratividade e geração de caixa inferiores às empresas nacionais. As diferenças de 6,2I pontos percentuais no caso de lucro líquido sobre vendas e 3,39 pontos percentuais também são significativas sob o aspecto gerencial. Por sua vez, empresas abertas, com ações na bolsa de valores, também apresentam melhor desempenho. Assim, a melhor situação seria a de uma empresa nacional com capital aberto. Esta empresa poderia ter uma vantagem combinada de II,76 pontos percentuais $(6,2 \mathrm{I}+5,55)$ de lucro líquido contra o caso extremo de uma empresa estrangeira de capital fechado. Não é possível, com esses dados, inferir causalidade, mas apenas a constatação do fato.

Finalmente, uma das variáveis de interesse da pesquisa, a produtividade, mostrou-se estatisticamente significativa em todas as regressões com coeficientes positivos, indicando uma relação positiva entre produtividade e desempenho financeiro. Empresas mais produtivas têm melhor desempenho. A regressão também permite dimensionar o grau dessa influência. O coeficiente de I,57 no caso do lucro líquido sobre vendas indica que, quando a variável produtividade assumir o valor I, ou seja, a produtividade for o dobro da média do ramo respectivo, a lucratividade será I,57 pontos percentuais superior. Intuitivamente, esse efeito pode ser considerado pequeno perante as demais fontes de variação e o desvio padrão total de I8,7 pontos percentuais para o lucro líquido sobre vendas. Isso coloca em questionamento a efetividade relativa dos esforços de busca de produtividade como mecanismo de melhora de desempenho financeiro.

A segunda variável de interesse, o tamanho da empresa, indicando o potencial de economias de escala nas operações das empresas de tecnologia de informação, não se mostrou estatisticamente significativa em nenhum dos casos. $O$ resultado sugere a inexistência de economias de escala e escopo líquidas que afetem o desempenho. 


\section{CONCLUSÕES}

Este estudo propôs-se a investigar a estrutura de variabilidade do desempenho financeiro das empresas do setor de tecnologia de informação e o papel de duas variáveis (tamanho e produtividade) que representam fatores operacionais nesse desempenho. A natureza dinâmica e evolutiva dos setores da nova economia poderia suportar que as empresas atuando nesses setores tivessem uma estrutura de variância de desempenho diferente, com maior influência do efeito ano e maior imprevisibilidade quanto ao desempenho. Os resultados não suportaram essa proposição e mostraram-se muito similares às demais empresas brasileiras e norte-americanas de outros setores econômicos relatados em estudos anteriores (MCGAHAN; PORTER, I997; HAWAWINI; SUBRAMANIAN; VERDIN, 2003; GONÇALVES; QUINTELLA, 2005). O maior fator foi sempre associado à empresa individual, o percentual de variância explicada foi equivalente aos estudos anteriores e o efeito ano foi, em geral, pequeno, não atingindo mais que I ou 2 pontos percentuais. Apenas no caso do crescimento o setor parece muito sensível ao ano, mas a ausência de estudos anteriores não permite uma comparação adequada com outros setores. Esses achados provocam um questionamento sobre as diferenças na análise de empresas atuantes na nova economia. Talvez os fatores sejam diferentes, mas o resultado final não parece ser.

Duas variáveis operacionais foram também investigadas. A variável tamanho não apresentou influência significativa no desempenho, seja este tomado como lucratividade ou crescimento. Essa constatação põe em dúvida a existência de economias de escala e escopo líquidas na área de tecnologia de informação, indicando que a área de operações dessas empresas não deveria se preocupar com elas. A variável produtividade apresentou uma relação positiva e estatisticamente significativa com o desempenho em todas as suas formas de medição. Empresas mais produtivas apresentam maior lucro, maior geração de caixa e maior crescimento que as demais. Esse resultado, consistente com a expectativa de operações, deve ser relativizado quando analisamos o grau dessa relação. Intuitivamente, a relação parece pequena e haveria a necessidade de grandes ganhos em produtividade para obter modestos ganhos em lucratividade e crescimento. Talvez os esforços da área de operações devessem buscar outros fatores de maior potencial de influência no desempenho. Eles certamente existem, dado o percentual de variância associado à empresa individual, mas produtividade não deve ser o primeiro da lista.

Esta última constatação também indica a necessidade de futuros estudos que identifiquem outras variáveis operacionais relevantes com potencial de influência no desempenho. Possivelmente, variáveis ligadas ao conhecimento, flexibilidade e inovação, sejam mais interessantes, explorando o conceito de capacidades dinâmicas (dynamic capabilities) (TEECE; PISANO; SHUEN, I997; EISENHARDT; MARTIN, 2000; WINTER, 2003). 
Esta pesquisa tem claras limitações que devem ser consideradas. A amostra é uma delas, dada a sua natureza não-probabilística já comentada. Mais variáveis deveriam ser exploradas, o que fica claramente evidenciado pelo percentual de variância explicada nas regressões que chegou, no máximo, a I7\%. Levantamento de dados primários seria o caminho necessário. $\mathrm{O}$ método de análise também tem limitações. O uso de regressões múltiplas tem como premissa a independência das observações entre si. Como estamos tratando de observações da mesma empresa, essa premissa está sendo violada. Apesar de isso ser uma abordagem comum em estudos de desempenho, as limitações existem. Uma solução seria o uso de técnicas multiníveis que poderiam ser usadas para modelar os dados longitudinais e ao mesmo tempo separar os diferentes níveis de análise e as variáveis para cada nível (HOFMANN, I997; RAUDENBUSH; BRYK, 2002; SINGER; WILLET, 2002).

\section{REFERÊNCIAS}

ANDERSON, E. W.; FORNELL, C.; RUST, R. T. Customer satisfaction, productivity, and profitability: differences between goods and services. Marketing Science, v. I6, n. 2, p. I29-I45, I997.

BANKER, R. D.; CHANG, H.-H.; MAJUNDAR, S. K. Analyzing the underlying dimensions of firm profitability. Managerial and Decision Economics, v. II4, n. I, p. 25-36, Jan. I993. BARNEY, J. B. Firm resources and sustained competitive advantage. Journal of Management, v. I7, n. I, p. 99-I20, I99I.

BARNEY, J. B.; ARIKAN, A. M. The resource-based view: origins and implications. In: HITT, M. A.; FREEMAN, R. E. et al. (Ed.). The Blackwell Handbook of Strategic Management. Oxford: Blackwell Publishers Ltd., 200I. p. I24-I88.

BESANKO, D. et al. Economics of strategy. 3. ed. Hoboken/NJ: John Wiley, 2004.

BRITO, L. A. L.; VASCONCELOS, F. C. Performance of Brazilian Companies: Year Effects, Line of Business, and Individual Firms. Brazilian Administration Review, v. I, n. I, p. I-I5, Jul./Dec. 2004.

BRUSH, T. H.; BROMILEY, P. What does a small corporate effect mean? A variance components simulation of corporate and business effects. Strategic Management Journal, v. I8, n. Io, p. 825835, 1997.

BRUSH, T. H.; BROMILEY, P.; HENDRICKX, M. The relative influence of industry and corporation on business segment performance: an alternative estimate. Strategic Management Journal, v. 20, n. 519-547, I999.

CAPON, N.; FARLEY, J. U.; HOENIG, S. Determinants of financial performance: a meta-analysis. Management Science, v. 36, n. I0, p. II43-II59, I990.

DAVIES, L. E.; GALLMAN R. E.; HUTCHINS, T. D. Technology, productivity, and profits: BritishAmerican Whaling Competition in the North Atlantic, I816-1842. Oxford Economic Papers, New Series, v. 39, n. 4, p. 738-759, Dec. I987. 
EASTERFIELD, T. E. Productivity - target or conceptual tool? OR, v. I6, n. 2, p. I77-I87, Jun. I965.

EISENHARDT, K. M.; MARTIN, J. A. Dynamic capabilities: what are they? Strategic Management Journal, v. 2I, n. IO-II, p. IIO5-II2I, 2000.

EXAME. As maiores empresas de tecnologia do Brasil. Exame, ano 20, n. 233, p. 76-ı18, 2005. GEROSKI, P. A.; MACHIN, S. J.; WALTERS, C. F. Corporate growth and profitability. The Journal of Industrial Economics, v. 45, n. 2, p. I7I-I89, I997.

GLICK, W. H.; WASHBURN, N. T.; MILLER, C. C. The myth of firm performance. Annual meeting of the Academy of Management. Honolulu, 2005. I4 p.

GONÇALVES, A. R.; QUINTELLA, R. H. Contribuição dos fatores internos e externos para o desempenho das empresas brasileiras e sua evolução na última década. In: XXIX EnANPAD. 2005. Brasília: Anpad, 2005. I8 p.

GOODING , R. Z.; WAGNER III, J. A. A meta-analytic review of the relationship between size and performance: the productivity and efficiency of organizations and their subunits. Administrative Science Quarterly, v. 30, n. 4, p. 462-48I, I985.

GUPTA, N. Some alternative definitions of size. Academy of Management Journal (pre-1986), v. 23, n. 4 , p. 759 , 1980.

HAWAWINI, G.; SUBRAMANIAN, V.; VERDIN, P. Is performance driven by industry-or firmspecific factors? A new look at the evidence. Strategic Management Journal, v. 24, n. I, p. I-I6, 2003. HENDERSON, R. M.; COCKBURN, I. M. Scale, scope and spillovers: the determinants of research productivity in drug discovery. RAND Journal of Economics, v. 27, n. I, p. 32-59, 1996.

HITT, L. M.; BRYNJOLFSSON, E. Productivity, business profitability, and consumer surplus: three different measures of information technology value. MIS Quarterly, v. 20, n. 2, p. I2I-I42, Jun. I996.

HOFMANN, D. A. An overview of the logic and rationale of hierarquical linear models. Journal of Management, v. 23, n. 6, p. 723-744, I997.

HOQUE, Z.; FALK, H. Industry characteristics and productivity measurement systems. International Journal of Operations \& Production Management, v. 20, n. II, p. I278-1292, 2000.

KERLINGER, F. N.; LEE, H. B. Foundations of behavioral research. 4. ed. Fort Worth/ TX: Harcourt College Publishers, 2000.

KIMBERLY, J. R. Organizational size and the structuralist perspective - a review, critique and proposal. Administrative Science Quarterly, v. 2I, n. 4, p. 57I, I976.

LA PORTA, R.; LOPEZ-DE-SILANES, F. The benefits of privatization. The Quarterly Journal of Economics, v. II4, n. 4, p. II93-I242, Nov. I999.

MCGAHAN, A.; PORTER, M. E. How much does industry matter, really? Strategic Management Journal, v. I8, n. 4, p. 15-30, I997.

MCNAMARA, G.; VAALER, P. M.; DEVERS, C. Same as it ever was: the search for evidence of increasing hypercompetition. Strategic Management Journal, v. 24, n. 3, p. 26I-278, 2003.

MCT. Política Nacional de Informática. 2003. Disponível em: <http://www.mct.gov.br/sepin/ pni/pni.htm>. Acesso em: i6 mar. 2006.

MDIC. Opções estratégicas - tecnologia de informação/semicondutores. 2004. Disponível em: <http:// www.desenvolvimento.gov.br/arquivo/ascom/imprensa/2004033IPlanoPoliticaIndustrial. pdf>. Acesso em: i6 mar. 2006. 
MILLER, D. M. Profitability = productivity + price recovery. Harvard Business Review, p. I45-I53, May/Jun. I984.

. Analyzing total factor productivity with ROI as criterion. Management Science, v. 33, n. II, p. I5OI-I505, I987.

MILLER, D. M; RAO, M. Analysis of profit-linked total factor productivity measurement models at the firm level. Management Science, v. 35, n. 6, p. 757-767, Nov. I989.

MISTEREK, S. Productivity as a performance measure. International Journal of Operations \& Production Management, v. I2, n. I, p. 29-45, 1992.

PENROSE, E. The theory of the growth of the firm. 3rd ed. Oxford/UK: Oxford University Press, I959.

PORTER, M. E. Competitive advantage: creating and sustaining superior performance. New York, London: Free Press/Collier Macmillan, I985.

What is strategy? Harvard Business Review, v. 74, n. 6, p. 6I-78, I996.

RAUDENBUSH, S. W.; BRYK, A. S. Hierarchical linear models: applications and data analysis methods. 2. ed. Thousand Oaks: Sage Publications, 2002.

RICKETTS, M. The economics of business enterprise. 3. ed. United Kingdom: Edward Elgar, 2002. ROQUEBERT, J. A.; PHILLIPS, R. L.; WESTFALL, P. A. Markets vs. management: what drives profitability? Strategic Management Journal, v . I7, p. 653-664, I996.

RUMELT, R. P. How much does industry matter? Strategic Management Journal, v. I2, n. 3, p. I67-I85, I991.

SCHMALENSEE, R. Do markets differ much? The American Economic Review, v. 75, n. 3, p. 34I35I, I985.

SEARLE, S. R.; CASELLA, G.; MCCULLOCH, C. E. Variance components. New York: John Wiley, I992.

SINGER, J. D.; WILLET, J. B. Applied longitudinal data analysis: modeling change and event occurrence. Oxford, New York: Oxford University Press, 2002.

SUTTON, J. Gibrat's legacy. Journal of Economic Literature, v. 35, p. 40-59, March I997.

TEECE, D. J.; PISANO, G. P.; SHUEN, A. Dynamic capabilities and strategic management. Strategic Management Journal, v. I8, n. 7, p. 509-533, I997.

WERNERFELT, B. A resource-based view of the firm. Strategic Management Journal, v. 5, n. 2, p. I7I-I80, I984.

WINTER, S. G. Understanding dynamic capabilities. Strategic Management Journal, v. 24, n. Io, p. 99I-995, 2003.

ZENGER, T. R. Explaining organizational diseconomies of scale in R\&D: agency problems and the allocation of engineering talent, ideas, and effort by firm size. Management Science, v. 40, n. 6, p. 708-729, 1994.

TRAMITAÇ ÃO

Recebido em 2/5/2007

Aprovado em 7/8/2007 